\title{
An Operational Land Imager for the Landsat Data Continuity Mission
}

\author{
James R. Irons \\ Biospheric Sciences Branch \\ NASA Goddard Space Flight Center \\ Greenbelt, Maryland, USA
}

\author{
Jeanine Murphy-Morris \\ Landsat Data Continuity Mission Project Office \\ NASA Goddard Space Flight Center \\ Greenbelt, Maryland, USA
}

\begin{abstract}
The National Aeronautics and Space Administration (NASA) released a request for proposals (RFP) on January 09, 2007 for an Operational Land Imager (OLI). The RFP specified the OLI as the principal sensor for the Landsat Data Continuity Mission (LDCM), the successor mission to Landsat 7. The RFP provides requirements for OLI performance, special calibration tests, and mission assurance without specifying a particular technical design. The performance specifications derive from the heritage and societal benefits of data from the previous Landsat satellites, the high standards set by the performance of the Landsat 7 Enhanced Thematic Mapper-Plus, direction from the Executive Office of the President, cognizance of current technology, and an eye to the future of land remote sensing beyond the LDCM. The specification includes nine spectral bands (four visible, one near infrared, three shortwave infrared, and one panchromatic band), spatial resolution of $30 \mathrm{~m}$ with the exception of a $15 \mathrm{~m}$ panchromatic resolution, stringent radiometric performance and absolute calibration, and accurate image geolocation with precise band-to-band and multitemporal image-to-image registration. Proposals were due on February 23, 2007 with selection and contract award anticipated by June 2007. The RFP requires delivery of a well-characterized instrument for spacecraft in tegration within 39 months of contract award. This schedule leads to a target LDCM launch date of July, 2011.
\end{abstract} Imager

Keywords-Landsat Data Continuity Mission; Operational Land

\section{INTRODUCTION}

The United States government agencies currently responsible for the Landsat program, the National Aeronautics and Space Administration (NASA) and the Department of Interior / United States Geological Survey (DOI / USGS) have now embarked on a third implementation strategy for a successor mission to Landsat 7. The two agencies initially attempted to implement a data procurement strategy, purchasing data from a privately-owned and commerciallyoperated satellite system developed through a partnership between the government and private industry. The successor mission was christened the Landsat Data Continuity Mission (LDCM) during this time. The data procurement strategy was terminated in September, 2003 when NASA declined to accept proposals submitted in response to a request for proposals (RFP) released in January, 2003 for a joint government / industry development of an LDCM satellite system. The U.S. government next tried to incorporate Landsat sensors onto satellite platforms in development for the National Polarorbiting Operational Environmental Satellite System (NPOESS). The United States government abandoned this implementation strategy when the technical complexities of incorporating Landsat sensors onto the multiple-sensor NPOESS satellites became apparent. A December 2005 memorandum from the United States Office of Science and Technology Policy (OSTP) directed NASA and the DOI / USGS to pursue the current strategy of launching a free-flying LDCM satellite.

The OSTP memorandum directed NASA to acquire a freeflyer satellite for collecting the required land surface data. The memorandum assigned DOI/USGS responsibility for operating the satellite following launch and on-orbit check-out and for collecting, archiving, processing, and distributing the land surface data. NASA responded by releasing a synopsis of its acquisition approach on February 22, 2006 and followed up with a revised synopsis on October 24,2006 . The revised synopsis remains valid and lays out a plan to acquire an instrument, spacecraft, and mission operations elements via separate procurements. The synopsis identifies NASA Goddard Space Flight Center (GSFC) as the system integrator for the overall mission with launch services acquired through the NASA Launch Services (NLS) contract managed by the Kennedy Space Center (KSC). NASA began calling the primary LDCM instrument the Operational Land Imager (OLI) and released an OLI RFP on January 09, 2007 as the first step in its acquisition plan.

\section{THE OPERATIONAL LAND IMAGER REQUEST FOR PROPOSALS}

The RFP solicits proposals for the delivery of an imaging sensor capable of meeting specified performance requirements on a cost reimbursable contract basis. The RFP document package includes a statement of work, performance requirements, special calibration test requirements, satellite interface requirements, and key performance requirements. The statement of work defines the contract deliverables with the major deliverable consisting of the OLI due within 39 months of contract award. The other requirements derive principally from the Landsat program mission and heritage. 
The complete RFP document package may be accessed from the NASA Acquisition Internet Service (NAIS) at the following internet address: http://prod.nais.nasa.gov/cgibin/eps/sol.cgi?acqid $=122610$. Proposals were due by February 23, 2007 and are currently under evaluation. Contract award is anticipated in June 2007.

The LDCM has a mandate to provide continuity in the multi-decadal Landsat land surface observations to study, predict, and understand the consequences of land surface dynamics. Both the mission name and the performance requirements in the RFP reflect this mandate. Irons and Masek [1] review the long and involved heritage that has led to the performance requirements. To summarize, Landsat satellites have collected multispectral, moderate resolution images of the global land surface for over 30 years with much of the data archived and available to the public from the DOI / USGS Earth Resources Observation and Science (EROS) facility in Sioux Falls, South Dakota. Landsat 7 is the most recent satellite in the series having been launched in 1999. The performance and operation of the Landsat 7 imaging sensor, the Enhanced Thematic Mapper-Plus (ETM+), set a standard for future Landsat missions. The Landsat 7 satellite with its ETM+ collects well-calibrated data affording seasonal coverage of the global land surface. Unfortunately, a failure of a component within the ETM+ optical system, the scan line corrector, has marred ETM+ data with periodic horizontal gaps in the images. In any case, the 1992 Land Remote Sensing Policy Act directed NASA and DOI / USGS to study options for a successor to Landsat 7 that provides for data continuity. Consequently, the LDCM performance specifications owe heritage to the earlier Landsat satellites and sensors, particularly the ETM+. Additionally, NASA conducted a technology demonstration mission called the Earth Observing1 (EO-1) satellite launched in 2000 . EO-1 carried an Advanced Land Imager (ALI) sensor intended to demonstrate technologies applicable to future Landsat missions. The OLI performance requirements are also shaped by ALI performance and by some of the challenges offered by the technology.

This heritage leads to OLI requirements for the collection of multispectal data for nine spectral bands: four visible, one near infrared, three shortwave infrared, and one panchromatic band. The required spatial resolution is $30 \mathrm{~m}$ for all spectral bands except for the $15 \mathrm{~m}$ resolution required for the panchromatic band. The OLI requirements further specify stringent radiometric performance and absolute calibration and accurate image geolocation with precise band-to-band registration. These requirements are quantified and described in much greater detail by the Requirements Document within the OLI RFP package.

In addition to performance requirements, the RFP package also contains a Special Calibration Test Requirements document that specifies pre-flight and on-orbit check-out tests to characterize OLI performance. The tests are not only intended to verify that specifications are met but also to characterize performance for OLI calibration and data product validation. The specified pre-flight tests will measure the OLI relative spectral response, radiometric response, and spatial response while characterizing the pixel-to-pixel uniformity in these responses. The pre-flight alignment of pixel lines-of-site is also required. Many of the pre-flight tests are impossible or more difficult in orbit and are yet fundamental to understanding in flight performance. The check-out tests specified for the on-orbit commissioning phase of the LDCM mission are intended to measure shifts in OLI performance and response due to launch or the space environment. Furthermore, the tests will transfer radiometric and line-of-site calibrations from the ground to space. The test requirements derive from lessons learned on earlier Landsat missions and the ALI and are expected to result in the best calibrated sensor of the Landsat series.

The understanding and specification of tests required to characterize OLI performance helped define a set of key performance requirements (KPR) to serve as on-orbit incentive fee criteria. A KPR document in the RFP package sets quantitative performance standards for both negative and positive incentive fee percentages. The areas of performance subject to assessment for incentive fees include signal-to-noise ratios, edge response slope, band-to-band registration accuracy, radiometric response stability, pixel-to-pixel uniformity, as well as other criteria. The DOI / USGS will assess performance in these areas on a monthly basis following the commissioning phase and will award fees accordingly.

\section{ADDITIONAL LDCM PROCUREMENTS}

NASA and the DOI / USGS will also procure most of the other elements of the LDCM satellite system from private industry. NASA is using its existing Rapid Spacecraft Acquisition contract, under GSFC's Rapid Spacecraft Development Office (RSDO), to acquire the LDCM spacecraft element. NASA has solicited accommodation studies from spacecraft vendors within the RSDO catalog. Studies will begin in May 2007 to address LDCM-unique requirements and develop initial designs. The studies will be followed in the Fall 2007 by a request for offers (RFO) to implement the LDCM observatory with contract award expected before the end of the calendar year. The selected vendor will be required to integrate the OLI onto their spacecraft and deliver this integrated observatory to the launch site.

NASA in coordination with the US Geological Survey (USGS) will additionally issue a separate RFP for the LDCM mission operations element (MOE). This procurement will provide all of the computer hardware and software necessary to operate the LDCM observatory in flight. The release of the MOE RFP is planned for no later than the fourth quarter of calendar year 2007 with contract award expected by the second quarter of calendar year 2008 .

The MOE will be delivered to an USGS-managed Mission Operations Center (MOC) and the USGS will competitively procure a flight operations team (FOT) to run the MOC. The USGS will further acquire the ground network element to install a primary ground receiving station at the USGS EROS and to secure supplemental data receiving capabilities. Finally, the USGS will procure an archive element to capture OLI data and plans to develop an OLI Image Processing Element in house. The USGS will integrate these multiple elements of the LDCM ground system into a coherent architecture. 


\section{CONCLUSION}

After a number of false starts the implementation of the successor mission to Landsat 7 has begun to move forward with the release of an RFP for the OLI, the primary sensor for the LDCM. The RFP specifies the OLI in terms of performance requirements that derive from a data continuity mandate, from performance standards established by the Landsat 7 ETM+, from awareness of technological advancements, and from recognition of the role of the LDCM within future earth observing capabilities. This first step in implementation moves the LDCM towards a target launch date of July 2011.

\section{REFERENCES}

[1] Irons, J.R., and J.G. Masek, "Requirements for a Landsat Data Continuity Mission," Photgrammetric Engineering \& Remote Sensing," vol. 72 , no. 10 , pp. 1102-1108, Oct. 2006 . 